\title{
ERPs Reflect Lexical Identification in Word Fragment Priming
}

\author{
Claudia K. Friedrich, Sonja A. Kotz, Angela D. Friederici, \\ and Thomas C. Gunter
}

\begin{abstract}
Behavioral evidence suggests that spoken word recognition involves the temporary activation of multiple entries in a listener's mental lexicon. This phenomenon can be demonstrated in cross-modal word fragment priming (CMWP). In CMWP, an auditory word fragment (prime) is immediately followed by a visual word or pseudoword (target). Experiment 1 investigated ERPs for targets presented in this paradigm. Half of the targets were congruent with the prime (e.g., in the primetarget pair: $A M-A M B O S S$ [anvil]), half were not (e.g., $A M-$ PENSUM [pensum]). Lexical entries of the congruent targets
\end{abstract}

\section{INTRODUCTION}

To understand a spoken word, the speech recognition system has to identify a single entry among thousands of other stored lexical representations. Current models of spoken word recognition assume that this process is enabled by the temporary activation of multiple lexical candidates. Competition among activated entries supports the selection of a proper candidate (see Cutler \& Clifton, 1999; Frauenfelder \& Floccia, 1999, for recent reviews). Evidence for multiple activation and competition is revealed by several behavioral studies (e.g., Connine, Titone, Deelmann, \& Blasko, 1997; Gow \& Gordon, 1995; McQueen, Norris \& Cutler, 1994; Marslen-Wilson, 1990; Zwitserlood, 1989).

The present study aimed at identifying neurophysiological correlates of lexical identification in the eventrelated brain potential (ERP). This was realized by a modified version of word form priming (see Zwitserlood, 1996 for an overview), namely, cross-modal word fragment priming (CMWP). Primes in CMWP are fragments taken from spoken words (e.g., fee taken from feel). Participants are engaged in a lexical decision task to visual targets that follow the primes. Prime-target pairs are either congruent (e.g., fee-feed) or incongruent (e.g., fee-name). In general, congruent words are responded to faster than incongruent words (Cooper, Cutler, \& Wales, 2002; Cutler \& van Donselaar, 2001;

Max Planck Institute for Human Cognitive and Brain Sciences should receive activation from the prime. Thus, lexical identification of these targets should be facilitated. An ERP effect named P350, two frontal negative ERP deflections, and the N400 were sensitive to prime-target congruency. In Experiment 2 , the relation of the formerly observed ERP effects to processes in a modality-independent mental lexicon was investigated by presenting primes visually. Only the P350 effect could be replicated across different fragment lengths. Therefore, the P350 is discussed as a correlate of lexical identification in a modality-independent mental lexicon.
Soto-Faraco, Sebastian-Galles, \& Cutler, 2001; Spinelli, Segui, \& Radeau, 2001; Marslen-Wilson, 1990). It is discussed that lexical identification of congruent targets is facilitated as a result of the activation from their respective primes.

Recently, competition has been tested in CMWP by Soto-Faraco et al. (2001). In this experiment, effects for partially incongruent targets (e.g., cabal-cabellera [hair]) were contrasted with completely incongruent targets (e.g., sol-cabellera). Partially incongruent targets elicited slower responses than completely incongruent targets. This delay has been interpreted as inhibition of partially incongruent candidates from completely congruent entries (e.g., caballero [gentleman]).

In neurophysiological research, lexical activation for visually presented words in lexical decision has been related to the N400 in the ERP (Holcomb, Grainger \& O'Rourke, 2002) and to the M350 in the magnetoencephalographic response of the brain (MEG; Pylkkänen, Stringfellow, \& Marantz, 2002). However, both studies used different neurophysiological methods and different experimental manipulations.

Holcomb et al. (2002) reported an enhanced amplitude of the N400 for words with many orthographic neighbors as compared to words with few orthographic neighbors. Orthographic neighborhood is a concept in visual word recognition, which refers to words of identical length that vary only by one letter (Coltheart, Davelaar, Jonasson, \& Besner, 1977). It is assumed that visual word presentation activates a word and its or- 
thographic neighbors. For example, mime and dome are activated when dime is presented. Thus, the presentation of words with many neighbors results in a greater number of activated lexical entries than the presentation of words with few neighbors. Holcomb and collaborators assume that the greater number of activated lexical entries is reflected in an enhanced N400 amplitude.

Pylkkänen and coworkers (2002) reported that the M350 peaks earlier for words with higher phonotactic probability than for words with lower phonotactic probability. Phonotactic probability refers to the frequency of individual sounds and sound sequences within a spoken language. For example, the phoneme sequence in the word fish has a lower phonotactic probability than the phoneme sequence in the word cat. It is proposed that words with high phonotactic probability are activated faster than words with low phonotactic probability (Vitevitch \& Luce, 1999). Accordingly, Pylkkänen and colleagues interpreted the modulated peak latency of the M350 as a reflection of the speed of lexical activation.

Given the different experimental manipulations, it is not completely clear whether the M350 and the N400 are manifestations of the same process. With respect to evidence in favor of a semantic interpretation of the N400, Holcomb and collaborators (2001) associated the N400 effect with increased activation at the level of semantic representations. In contrast, the M350 appears to be more closely related to activation at the level of phonological representations. Furthermore, an enhanced N400 for words with many orthographic neighbors may not necessarily be related to lexical activation, but may reflect inhibition: A word with many neighbors receives more inhibition from activated competitors than a word with few neighbors. However, this interpretation is contrary to the report on facilitated reaction times found for words with many orthographic neighbors (e.g., Andrews, 1989). Therefore, the present study attempts to reveal additional insights into the timing of lexical activation and influences of the number of simultaneously activated candidates in the ERP.

In Experiment 1, we recorded ERPs for congruent targets in prime-target pairs like am-Amboss [anvil] and for incongruent targets in pairs like am-Pensum [pensum]. Primes should activate congruent targets, but not incongruent ones. Thus, an ERP correlate of lexical identification should differentiate both types of targets. To explore effects of simultaneously activated competitors, we manipulated the length of the fragments. For example, am is congruent with words like Amboss, Amber [amber] or Ampel [traffic light], whereas a longer fragment such as amb is only completely congruent with words like Amboss and Amber, but not with Ampel.

The point at which a fragment is congruent to only one word, is referred to as the isolation point (IP). We determined this point for each congruent target word in a separate gating experiment during which successive auditory fragments were presented (see Grosjean, 1996, for a detailed introduction to this paradigm). Twenty subjects that did not participate in the current study were asked to predict-after the presentation of each fragment-the word they thought would be presented. The IP was reached when all participants correctly produced the target word.

Three types of fragments were created in relation to the IP. Long fragments were fragments up to the IP, which could only be completed by the target word (e.g., ambo). Medium fragments were derived from the long fragments by subtracting one phoneme (e.g., $a m b$ ). Short fragments were derived by subtracting two phonemes from the long fragments (e.g., am). For medium as well as for short fragments, a group of matching entries next to the congruent target word exists, although, this group is smaller for medium fragments than for short fragments. The smaller the amount of activated competitors, the less inhibition a congruent target should receive.

In Experiment 2, we tested whether ERP correlates of CMWP could also be elicited in a unimodal priming paradigm. With respect to CMWP, it is assumed that identification occurs in a modality-independent mental lexicon (see Marslen-Wilson, 1990). Thus, it should be possible to replicate ERP effects related to lexical identification in CMWP by using visual fragments followed by visual targets in a unimodal paradigm (see Figure 1 for illustration of both paradigms).

In both experiments, subjects were engaged in a lexical decision task. It is known from priming that responses in the lexical decision task do not only reflect lexical processes, but also strategic aspects such as expectancy-induced priming (e.g., Posner \& Snyder, 1975) and prime-target matching mechanisms (e.g., Norris, McQueen \& Cutler, 2002; Neely, Keefe, \& Ross, 1989; Radeau, Morais, \& Dewier, 1989). Expectancyinduced priming refers to a strategy subjects use to

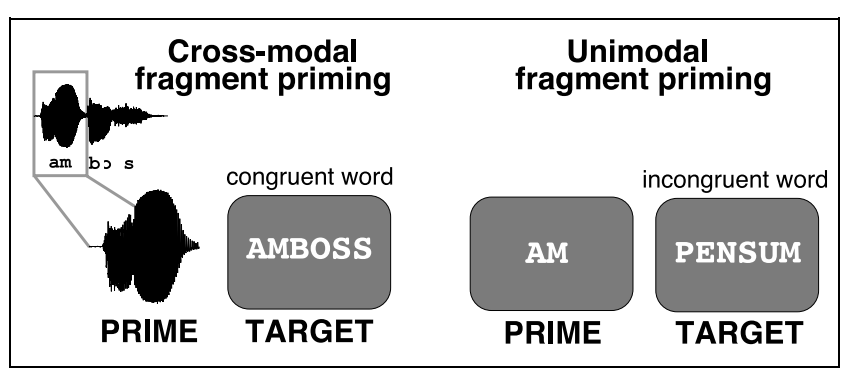

Figure 1. Illustration of one trial in cross-modal fragment priming (left) and unimodal fragment priming (right). In cross-modal priming, fragments were taken from spoken words as illustrated for am taken from Amboss. In unimodal priming, fragments were presented visually for $200 \mathrm{msec}$. In both experiments, visual targets were presented for $200 \mathrm{msec}$ immediately following the primes. Targets as well as visual primes were presented in capital letters using COURIER font. 
generate an expectancy set of words from the prime. If a target word is included in the expectancy set, it is responded to faster than if it is not included. The probability that expectancy contributes to the priming effect increases with a large portion of related target words (Keefe \& Neely, 1990). To minimize benefits of expectancy, pseudowords that differ only in the last one or two letters from the words were created.

Prime-target matching refers to a bias in lexical decision, which is caused by the yes-no response alternative. Subjects are assumed to match primes and targets following lexical identification of a target. If there is a relation between both, "yes" responses are facilitated. To control for response congruency bias, we compared congruency effects for words and pseudowords in both experiments. If a facilitation for target words originates from response congruency, a reversed effect should be observed for pseudowords, which require a "no" response.

\section{RESULTS: EXPERIMENT 1}

\section{Behavioral Measures}

The top of Figure 1 illustrates behavioral data observed in CMWP. Three-way analyses of variance (ANOVAs) across subjects $\left(F_{1}\right)$ and across items $\left(F_{2}\right)$ including the factors Wordness (words vs. pseudowords), Congruency (congruent targets vs. incongruent targets), and Fragment length (short fragments vs. medium fragments vs. long fragments) were conducted. There were significant main effects of the factors Wordness $\left[F_{1}(1,23)=113.45\right.$, $\left.p<.001 ; F_{2}(1,107)=181.45, p<.001\right]$, Congruency $\left[F_{1}(1,23)=279.46, p<.001 ; F_{2}(1,107)=94.88, p<\right.$ $.001]$, and Fragment length $\left[F_{1}(2,46)=31.11, p<.001\right.$; $\left.F_{2}(2,214)=15.66, p<.001\right]$. Furthermore, there were significant interactions between the factors Wordness and Congruency $\left[F_{1}(1,23)=80.39, p<.001 ; F_{2}(1,107)=\right.$ $71.50, p<.001]$, and Congruency and Fragment length $\left[F_{1}(2,46)=23.45, p<.001 ; F_{2}(2,214)=20.02, p<.001\right]$.

Facilitation for congruent targets was greater for words $\left[F_{1}(1,23)=280.31, p<.001 ; F_{2}(1,107)=\right.$ 132.97, $p<.001]$, than for pseudowords $\left[F_{1}(1,23)=\right.$ $\left.11.76, p<.01 ; F_{2}(1,107)=5.83, p=.02\right]$. Although facilitation for congruent targets was significant across all three prime lengths [short: $F_{1}(1,23)=57.37, p<$ $.001, F_{2}(1,107)=27.86, p<.001 ;$ medium: $F_{1}(1,23)=$ 98.42, $p<.001, F_{2}(1,107)=46.03, p<.001 ;$ long: $F_{1}(1,23)=166.53, p<.001, F_{2}(1,107)=156.09, p<$ $.001]$, the effect increased with increasing fragment length. The difference of RTs for congruent targets and incongruent targets was $15 \mathrm{msec}$ larger for medium as compared to short fragments and $31 \mathrm{msec}$ larger for long as compared to medium fragments (see top of Figure 2A).

For error rates, there were significant main effects of the factors Wordness $\left[F_{1}(1,23)=32.25, p<.001\right.$;
$\left.F_{2}(1,107)=15.02, p=.02\right]$ and Congruency $\left[F_{1}(1,23)\right.$ $\left.=65.44, p<.001 ; F_{2}(1,207)=22.27, p<.001\right]$, which were qualified by a significant interaction of both factors $\left[F_{1}(1,23)=108.86, p<.001 ; F_{2}(1,107)=60.19, p<\right.$ $.001]$. Error rates were lower for congruent words than for incongruent words $\left[S D=9, F_{1}(1,23)=103.93, p<\right.$ $\left..001 ; F_{2}(1,207)=60.05, p<.001\right]$, but higher for congruent pseudowords than for incongruent pseudowords $\left[F_{1}(1,23)=26.66, p<.001 ; F_{2}(1,207)=5.32\right.$, $p=.02$; see top of Figure 2B].

\section{ERP Measures}

ERPs for congruent and incongruent targets started to differ 200 msec after target onset (see Figures 3A and 4A). Incongruent targets elicited more positive amplitudes than congruent targets irrespective of wordness. The corresponding ERP deflection-ranging from 200 to $400 \mathrm{msec}$ with a peak at $350 \mathrm{msec}$ and a posterior-left scalp distribution (see Figure 8A) - is referred to as P350. Between 300 and $500 \mathrm{msec}$, incongruent words elicited a larger negativity over the right anterior scalp region than congruent words (right frontal negativity, see Figure 3A). In contrast, congruent pseudowords elicited a larger negativity over the left anterior scalp region than incongruent pseudowords (left frontal negativity, see Figure 4A). Furthermore, there was an N400 component ranging from 400 to $600 \mathrm{msec}$, which showed an enhanced amplitude for incongruent as compared to congruent words, but did not differ for incongruent and congruent pseudowords. Three different time windows (200 to $400 \mathrm{msec}$ for the P350, 300 to $500 \mathrm{msec}$ for the anterior ERP deflections, and 400 to $600 \mathrm{msec}$ for the N400) were statistically analyzed with five-way ANOVAs, which included the factors Wordness, Congruency, and

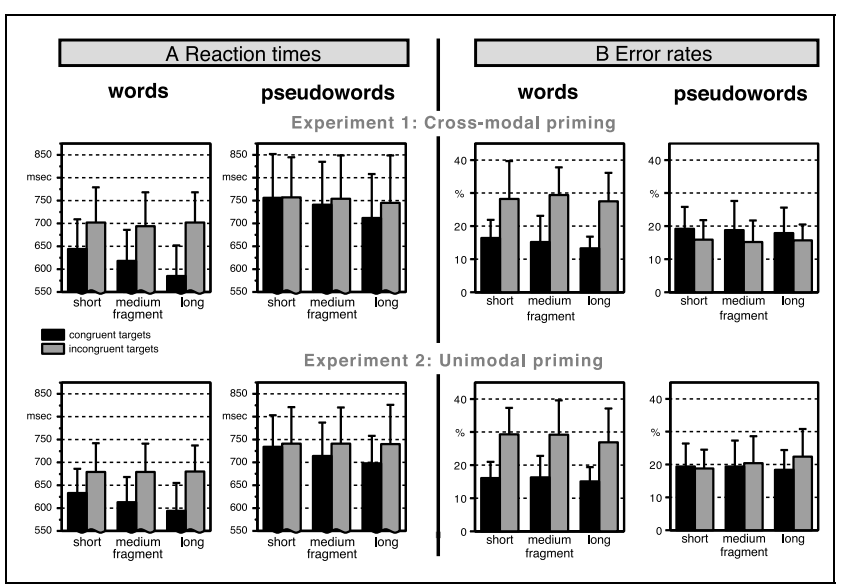

Figure 2. (A) Reaction times and (B) errors for congruent targets (black) and incongruent targets (gray) for words (left) and pseudowords (right) following short, medium, and long primes in cross-modal word fragment priming (above) and in unimodal fragment priming (below). 


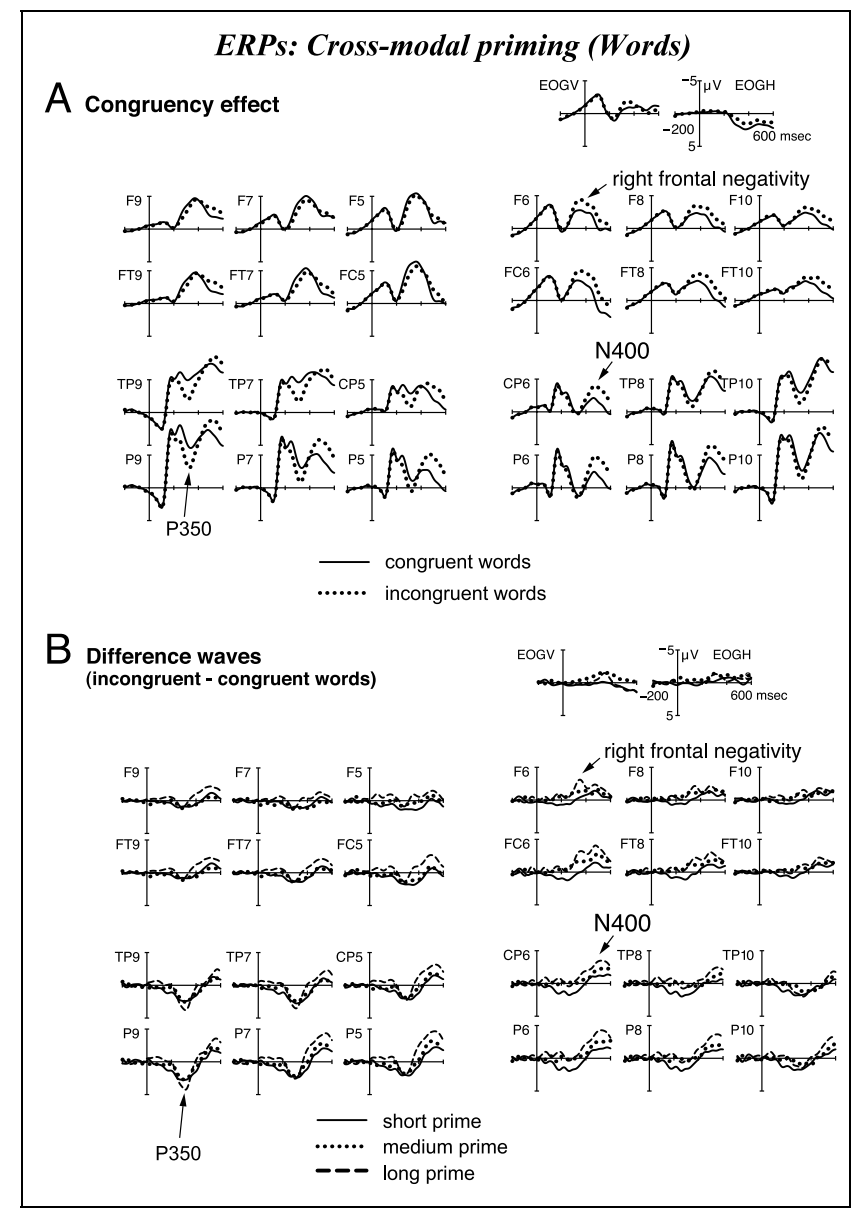

Figure 3. (A) ERPs at selected electrode sites elicited by congruent words (solid line) and by incongruent words (dotted line) across all prime lengths in Experiment 1 (cross-modal priming). (B) Difference waves representing amplitude differences (incongruent wordscongruent words) for short primes (solid line), medium primes (dotted line), and long primes (dashed line). Incongruent words elicited a larger P350 and a larger N400 than congruent words.

Fragment length, as well as Hemisphere (left vs. right) and Region (anterior vs. posterior).

\section{0 to $400 \mathrm{msec}$}

Statistical analysis revealed significant interactions of the factors Wordness and Region $[F(1,23)=28.01, p<$ $.001]$, Wordness, Region, and Hemisphere $[F(1,23)=$ $4.40, p=.05]$, Congruency and Region $[F(1,23)=21.78$, $p<.001]$, Congruency and Hemisphere $[F(1,23)=$ $40.71, p<.001]$, and Congruency, Hemisphere, and Region $[F(1,23)=4.61, p=.04]$.

In the first time window we focused on the posteriorly left distributed P350 effect. This ERP deflection seemed enhanced for pseudowords as compared to words (see Figure 7A). However, there was only a marginal effect in the posterior left region of interest $[\mathrm{ROI} ; F(1,23)=3.34$, $p=.07]$. Furthermore, the amplitude of the P350 was enhanced for incongruent targets as compared to con- gruent targets, which was supported by significant post hoc comparisons for the posterior left ROI $[F(1,23)=$ $25.11, p<.001]$ and the anterior left ROI $[F(1,23)=$ $5.13, p=.03$; see Figures $3 \mathrm{~A}$ and $4 \mathrm{~A}$, and Figure $8 \mathrm{~A}$ for the scalp distribution].

\section{0 to $500 \mathrm{msec}$}

The ANOVA for the second time window revealed a significant main effect of the factor Wordness $[F(1,23)=$ $10.97, p<.01]$ and significant interactions of the factors Wordness and Region $[F(1,23)=41.13, p<.001]$, Wordness and Congruency $[F(1,23)=10.49, p<.01]$, and Congruency, Region, and Hemisphere $[F(1,23)=$ $24.70, p<.001]$.

In the second time window, we focused on anterior negative ERP deflections. Post hoc comparisons for the anterior scalp region revealed enhanced amplitudes for

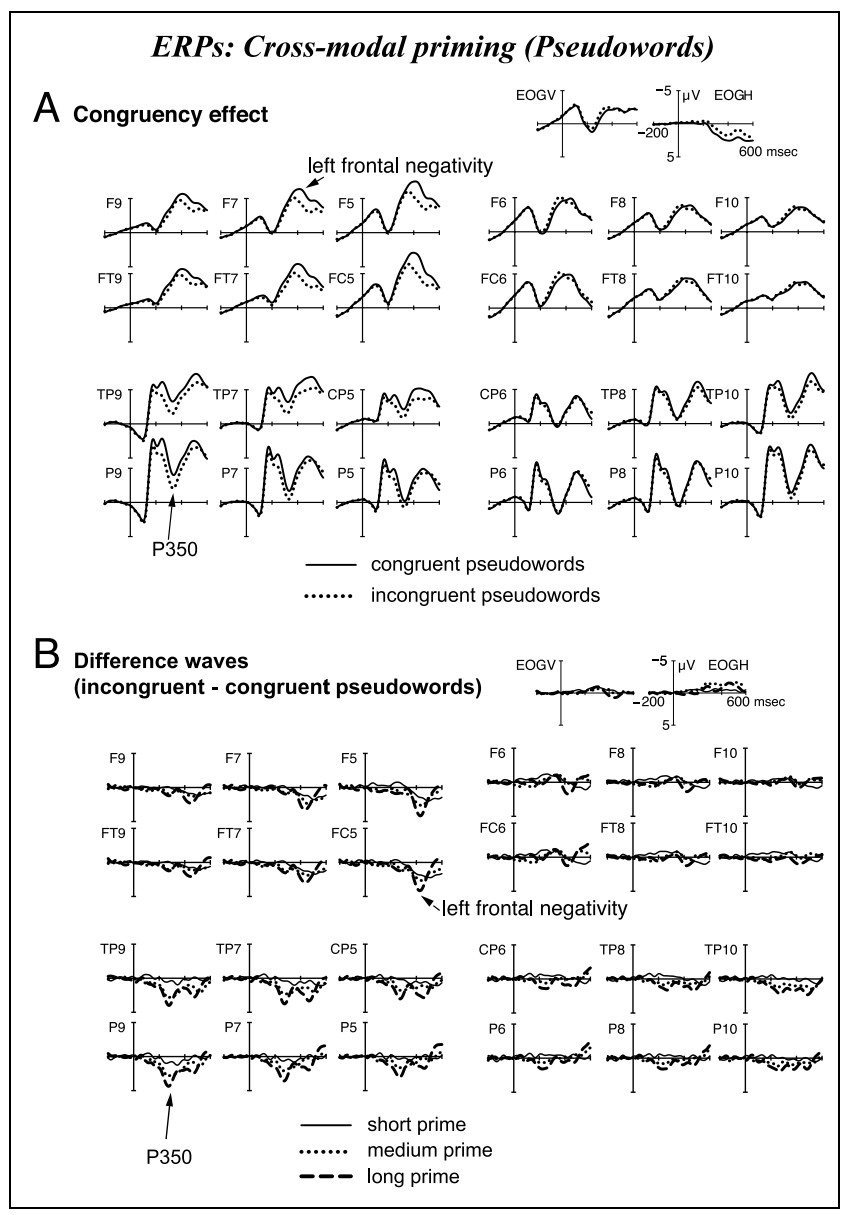

Figure 4. (A) ERPs at selected electrode sites elicited by congruent pseudowords (solid line) and by incongruent pseudowords (dotted line) across all prime lengths in Experiment 1 (cross-modal priming). (B) Difference waves representing amplitude differences (incongruent pseudowords-congruent pseudowords) for short primes (solid line), medium primes (dotted line), and long primes (dashed line). Incongruent pseudowords elicited a larger P350 and a reduced left frontal negativity than congruent pseudowords. 
pseudowords as compared to words $[F(1,23)=34.70$, $p<.001$, see Figure 7A]. Across words and pseudowords, congruent targets elicited a larger negativity than incongruent targets over the anterior left ROI $[F(1,23)$ $=6.37, p=.02]$. However, as can be seen in Figure 4A, this left anterior negativity can only be observed for pseudowords $[F(1,23)=4.41, p=.05]$. The reversed pattern was found over the right anterior ROI, where a larger negativity for incongruent as compared to congruent targets was elicited $[F(1,23)=7.45, p=.01]$. This right frontal negativity was only found for words $[F(1,23)=3.34, p=.08$, see Figure $3 \mathrm{~A}]$.

\section{0 to $600 \mathrm{msec}$}

There were significant interactions between the factors Wordness and Congruency $[F(1,23)=25.48, p<.001]$, Congruency and Hemisphere $[F(1,23)=7.77, p=.001]$, Wordness, Congruency, and Hemisphere $[F(1,23)=$ $14.62, p<.001]$, and Congruency, Hemisphere, and Region $[F(1,23)=21.85, p<.001]$.

In the third time window, we focused on the posteriorly distributed $\mathrm{N} 400$, which showed a significantly enhanced amplitude for incongruent targets as compared to congruent targets over the right posterior ROI $[F(1,23)=7.57, p=.01]$ and over the left posterior ROI $[F(1,23)=5.18, p=.03$, see Figures $3 \mathrm{~A}$ and $4 \mathrm{~A}]$. However, congruency effects differed for words and pseudowords. The N400 effect was found for words in both hemispheres [left: $F(1,23)=9.61, p<.01$; right: $F(1,23)=2.81, p<.001$; see Figure $3 \mathrm{~A}]$, but not for pseudowords (see Figure 4A).

\section{Summary of Experiment 1 and Motivation for Experiment 2}

Taken together, four ERP deflections are related to target processing in CMWP. We found a positive-going ERP deflection with a peak at $350 \mathrm{msec}$, named P350. The amplitude of the P350 was enhanced for incongruent targets as compared to congruent targets. The P350 effect occurred independently of word status. Furthermore, negative ERP deflections peaking at $400 \mathrm{msec}$ were elicited over the frontal scalp region. The amplitude of a right frontal negativity was larger for incongruent words than for congruent words, whereas the amplitude of a left frontal negativity was larger for congruent pseudowords as compared to incongruent pseudowords. Finally, the amplitude of the N400 was found to be enlarged for incongruent words as compared to congruent words, but not for incongruent pseudowords as compared to congruent pseudowords. Although the behavioral facilitation did clearly improve with fragment length, the ERP failed to reveal significant interactions with this factor.

Given the present ERP results, it seems that more than one process is tapped with CMWP. The question remains which of the ERP deflections may be related to lexical identification in a modality-independent mental lexicon. Therefore, Experiment 2 investigates which of the ERP deflections observed in Experiment 1 can also be elicited when primes are presented in the visual modality.

\section{RESULTS: EXPERIMENT 2}

\section{Behavioral Measures}

The bottom of Figure 2 illustrates behavioral data in unimodal fragment priming. For the reaction times, there were significant main effects of the factors Wordness $\left[F_{1}(1,23)=133.36, p<.001 ; F_{2}(1,107)=195.27\right.$, $p<.001]$, Congruency $\left[F_{1}(1,23)=100.37, p<.001\right.$; $\left.F_{2}(1,107)=118.10, p<.001\right]$, and Fragment length $\left[F_{1}(2,46)=15.01, p<.001 ; F_{2}(2,214)=16.11, p<\right.$ .001]. Main effects were qualified by interactions of the factors Wordness and Congruency $\left[F_{1}(1,23)=37.19, p<\right.$ $\left..001 ; F_{2}(1,107)=31.73, p<.001\right]$, and Congruency and Fragment length $\left[F_{1}(2,46)=22.56, p<.001 ; F_{2}(2,214)=\right.$ $14.56, p<.001]$.

Facilitation for congruent targets was greater for words $\left[F_{1}(1,23)=135.89, p<.001 ; F_{2}(1,107)=119.42\right.$, $p<.001]$ than for pseudowords $\left[F_{1}(1,23)=19.58\right.$, $\left.p<.01 ; F_{2}(1,107)=16.53, p<.001\right]$. Again, there was a significant facilitation for congruent targets across all three prime lengths [short: $F_{1}(1,23)=23.73, p<.001$, $F_{2}(1,107)=29.62, p<.001 ;$ medium: $F_{1}(1,23)=121.62$, $p<.001, F_{2}(1,107)=72.66, p<.001 ;$ long: $F_{1}(1,23)=$ $\left.91.79, p<.001, F_{2}(1,107)=159.29, p<.001\right]$, which increased with fragment length. There was a facilitation of $19 \mathrm{msec}$ from short to medium fragments and a facilitation of $17 \mathrm{msec}$ from medium to long fragments (see bottom of Figure 2A).

For the error rates, there was a significant main effect of the factor Congruency $\left[F_{1}(1,23)=142.28, p<.001\right.$; $\left.F_{2}(1,207)=39.74, p<.001\right]$, which was qualified by a significant interaction of the factors Wordness and Congruency $\left[F_{1}(1,23)=56.46, p<.001 ; F_{2}(1,107)=\right.$ $29.92, p<.001]$. Error rates were lower for congruent words than for incongruent words $\left[F_{1}(1,23)=125.20\right.$, $\left.p<.001 ; F_{2}(1,207)=55.02, p<.001\right]$. However, there was no significant difference between congruent pseudowords and incongruent pseudowords $\left[F_{1}(1,23)=\right.$ $3.83, p=.06 ; F_{2}(1,207)=1.06, n s$; see bottom of Figure 2B].

\section{ERP Measures}

In unimodal fragment priming, we replicated a P350 effect differentiating congruent and incongruent targets (see Figures 5A and 6A), and an N400 effect differentiating congruent and incongruent words (see Figure 5A). We did not find frontal effects for congruent and incongruent targets that were reported in Experiment 1 (compare Figures 3, 4, 5, and 6). To ensure comparabil- 


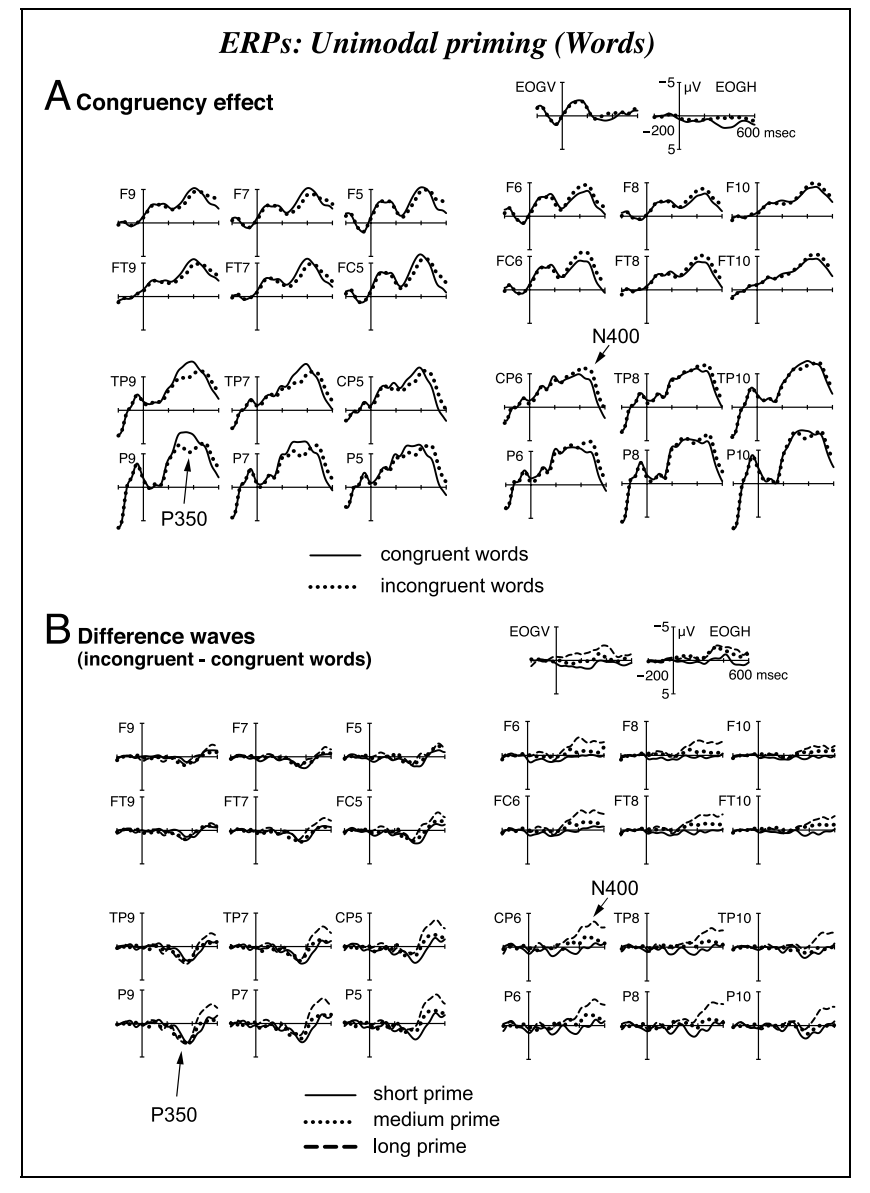

Figure 5. (A) ERPs at selected electrode sites elicited by congruent words (solid line) and by incongruent words (dotted line) across all prime lengths in Experiment 2 (unimodal priming). (B) Difference waves representing amplitude differences (incongruent wordscongruent words) for short primes (solid line), medium primes (dotted line), and long primes (dashed line). Incongruent words elicited a larger P350 than congruent words. An enhanced amplitude of the N400 for incongruent as compared to congruent words could only be proofed for long primes.

ity between both experiments, we analyzed the same three time windows as in Experiment 1 with similar ANOVAs and post hoc analyses.

\section{0 to $400 \mathrm{msec}$}

There was a significant interaction of the factors Congruency and Hemisphere $[F(1,23)=34.14, p<.001]$ in the first time window. Post hoc comparisons indicated a larger amplitude of the P350 for incongruent targets as compared to congruent targets over the left hemisphere $[F(1,23)=5.72, p=.03$; see Figures $5 \mathrm{~A}$ and $6 \mathrm{~A}$, and Figure $8 \mathrm{~B}$ for the scalp distribution].

\section{0 to $500 \mathrm{msec}$}

In the second time window, there was a significant effect of the factor Wordness $[F(1,23)=10.42, p<.01]$, which was modulated by a significant interaction of the factors Wordness and Region $[F(1,23)=6.44, p=.02]$. Pseudowords elicited a larger frontal negativity than words $[F(1,23)=20.24, p<.001$; see Figure $7 \mathrm{~B}]$.

\section{0 to $600 \mathrm{msec}$}

There was a significant main effect of the factor Wordness $[F(1,23)=63.12, p<.001]$ and significant interactions of the factors Wordness and Congruency $[F(2,46)$ $=6.53, p=.02]$, Congruency and Fragment length $[F(2,46)=3.84, p=.03]$, Congruency, Fragment length, and Region $[F(2,46)=5.53, p<.01]$, Congruency, Hemisphere, and Region $[F(1,23)=7.07, p=.01]$, and Congruency, Fragment length, Hemisphere, and Region $[F(2,46)=4.21, p=.02]$.

Pseudowords elicited a larger amplitude of the N400 than words (see Figure 7B), but a main effect of the

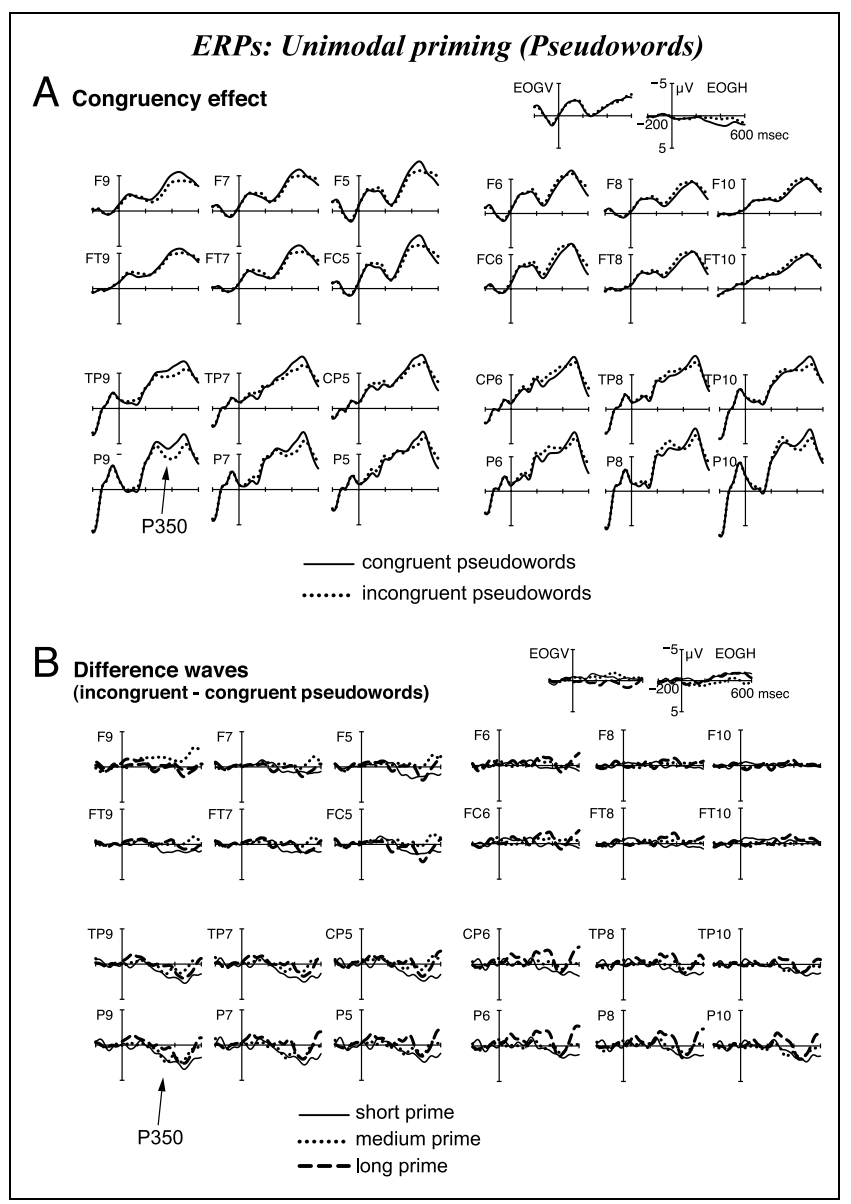

Figure 6. (A) ERPs at selected electrode sites elicited by congruent pseudowords (solid line) and by incongruent pseudowords (dotted line) across all prime lengths in Experiment 2 (unimodal priming). (B) Difference waves representing amplitude differences (incongruent pseudowords-congruent pseudowords) for short primes (solid line), medium primes (dotted line), and long primes (dashed line). Incongruent pseudowords elicited a larger P350 and a reduced left frontal negativity as compared to congruent pseudowords. 


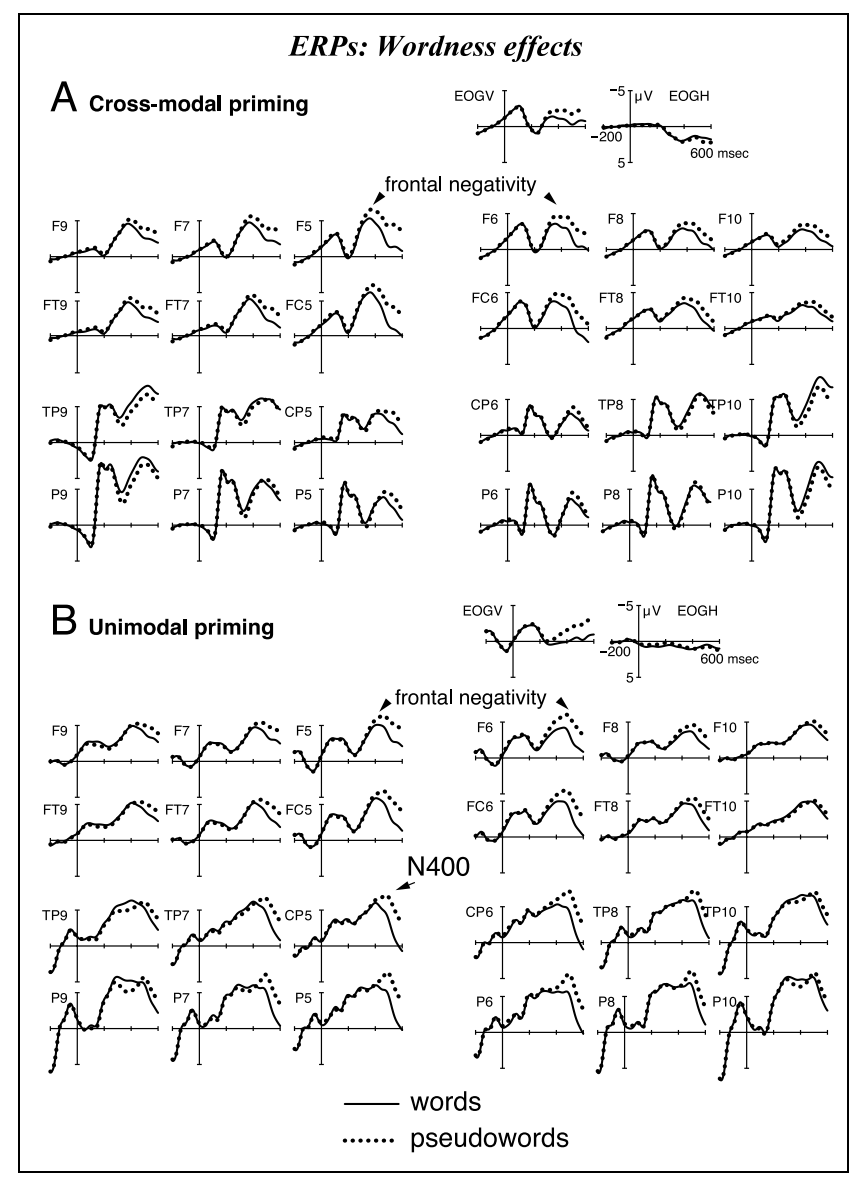

Figure 7. ERPs at selected electrode sites elicited by words (solid line) and by pseudowords (dotted line) across all prime lengths (A) in Experiment 1 (cross-modal priming), and (B) in Experiment 2 (unimodal priming). Pseudowords elicited a larger frontal negativity across both experiments, and a larger N400 in unimodal priming. factor Congruency could only be found for words $[F(1,23)=6.06, p=.02]$. However, this effect was modulated by fragment length. A larger N400 for incongruent words as compared to congruent words was only observed for long fragments over the posterior left ROI $[F(1,23)=7.06, p=.01]$, and over the posterior right ROI $[F(1,23)=7.53, p=.01]$. There was no N400 effect related to congruency for words following short and medium fragments.

\section{DISCUSSION}

In the present study, we explored ERP correlates of lexical identification in CMWP. Congruency between primes and targets was manipulated to investigate lexical activation. Fragment length was modulated to explore effects of activated competitors. We found a P350 deflection, a right and a left frontal negativity, and the N400 to be sensitive to prime-target congruency in CMWP. In Experiment 2, we investigated which of these
ERP deflections could be replicated in unimodal fragment priming. Only the P350 effect did reliably differentiate congruent and incongruent targets in unimodal fragment priming. Neither frontal negative effects nor an N400 effect across all fragment lengths was observed in Experiment 2.

In both experiments, the reduced amplitude of the P350 for congruent as compared to incongruent targets was correlated with faster reactions to congruent targets. This behavioral facilitation replicates previous results observed for congruent target words in CMWP (Cooper et al. 2002; Cutler \& van Donselaar, 2001; Soto-Faraco et al., 2001; Spinelli et al., 2001; Marslen-Wilson 1990). So far, facilitated reactions for target words in CMWP have been interpreted as reflecting activation of congruent lexical entries. However, the finding that several ERP deflections differentiate congruent and incongruent targets challenge such an assumption. In the following, we will discuss possible processes underlying the ERP deflections observed in CMWP.

We argued in the introduction that an ERP effect related to lexical identification should differentiate congruent and incongruent target words. The P350 was the only effect for which this assumption was substantiated across different fragment lengths in both experiments. However, although the P350 might be a promising candidate to reflect lexical identification in a modalityindependent mental lexicon, parallel P350 effects for words and pseudowords question this interpretation. A possible explanation for this similarity is that the pseudowords were very close to the words, because the difference resulted from exchanging the last one or two letters only. Thus, the deviation point was always after the uniqueness point of the corresponding word.

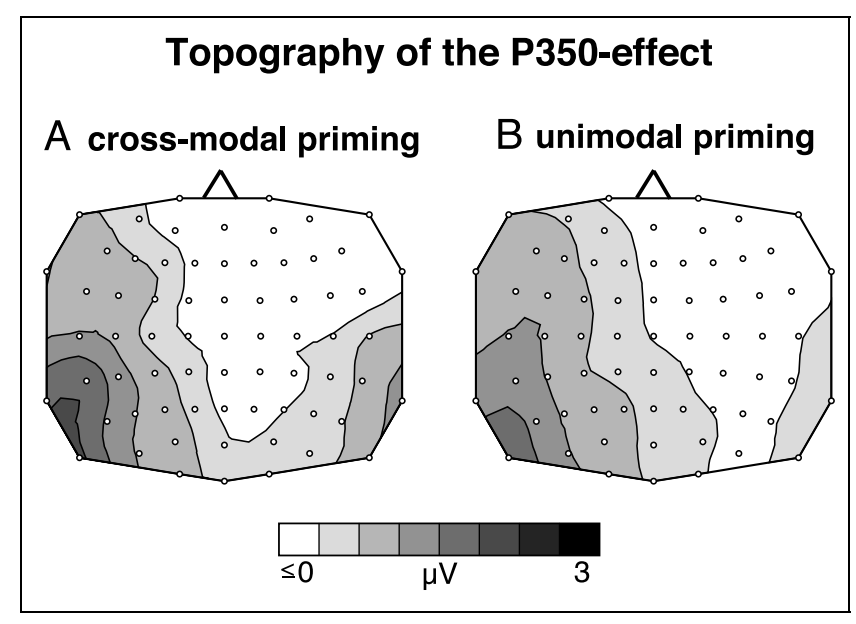

Figure 8. Topography of the $\mathrm{P} 350$ effect illustrated in difference maps (incongruent words-congruent words) in a time window ranging from 200 to $400 \mathrm{msec}$, (A) for Experiment 1 (cross-modal priming), and (B) for Experiment 2 (unimodal priming). Across both experiments, the P350 effect was pronounced over the left hemisphere with a maximum over temporal electrode sites. 
Following recent behavioral evidence for sequential aspects of visual word recognition (Lindell, Nichols \& Castles, 2003; Kwantes \& Mewhort, 1999), pseudowords might activate the same entries as words as long as no violation is detected. Accordingly, equal congruency effects for words and pseudowords in the amplitude of the P350 and in the reaction times might indeed be attributed to the same process, namely, lexical identification in a modality-independent mental lexicon.

The P350 temporally coincides with the M350 in the MEG, which has also been related to lexical activation during lexical decision (Pylkkänen et al., 2002). Both neurophysiological correlates show a comparable peak latency. Furthermore, the left hemispheric scalp distribution of the $\mathrm{P} 350$ resembles the source localization of the M350, which revealed an origin of that component in the left temporal cortex. However, although the P350 parallels the M350 in its latency and scalp distribution, both deflections differ in terms of how they were induced. The P350 varied in amplitude for congruent and incongruent targets, whereas the M350 was found to vary in peak latency for words with frequent and infrequent sound sequences. Variation in latency on the one hand and variation in amplitude on the other hand may be attributed to the different experimental manipulation in both studies. In the MEG experiment, phonotactic probability, which is thought to facilitate lexical identification, was varied. Therefore, we may speculate that speeded identification modulates the latency of the M350, whereas the reduced effort of lexical identification due to priming is reflected in a reduced amplitude of the $\mathrm{P} 350$.

The present data do not support the notion that the N400 effect observed when number of orthographic neighborhood is varied (Holcomb et al., 2002) is related to lexical activation. We may conclude that there are differences in the neurophysiological response to activation at the phonological level, which was tapped into in the present experiment, and the semantic level in the study by Holcomb and collaborators. Following this, the P350 may indicate identification of phonological representations, whereas the $\mathrm{N} 400$ may be related to activation of semantic representations. However, the possibility remains that the N400 effect observed by Holcomb and coworkers is related to competition rather than to activation.

Taken together, the present data point to an interpretation of the P350 as related to lexical identification in a modality-independent mental lexicon. An important question is why a P350 effect has not been previously reported in the ERP priming literature. The answer to that question probably lies in the specific paradigm we applied in the present study. Rather than using complete words as primes, we presented word fragments. Most likely, this novel aspect in ERP research has caused the elicitation of a previously undetected languagerelated ERP deflection, the $\mathrm{P} 350$. This ERP deflection appears to be a promising tool to investigate psycholinguistic hypotheses regarding the identification of spoken words with ERPs.

Similar P350 and reaction time effects for words and pseudowords rule out alternative explanations of priming effects in terms of strategic processes. It has been suggested that such processes check the relatedness between prime and target or prepare yes/no responses in the lexical decision task (e.g., Neely, 1991). If such processes would be reflected in the P350, we should have observed different effects for words and pseudowords. However, after a shared initial activation stage, words and pseudowords may be processed differently as revealed by different ERP effects for words and pseudowords following the P350. There were frontal negative ERP deflections and N400 effects differentiating words and pseudowords.

Both the right and the left frontal negativity, which were exclusively found in CMWP, suggest that more processes are tapped with CMWP than with unimodal fragment priming. We suggest that this difference originates from differences in auditory and visual word processing. A written word is presented as a whole, whereas, a spoken word is of sequential nature and thus is not completely available during the onset of the signal. Given this difference, spoken word recognition might be more strongly based on expectancy mechanisms regarding the upcoming word than visual word processing. It has been assumed that ERP effects for spoken word recognition in sentence context differ from that of visual word recognition in sentence context in an N200 effect preceding the N400 (van den Brink, Brown, \& Hagoort, 2001; Hagoort \& Brown, 2000). The amplitude of the N200 was found to be reduced for words that do not match a sentence context, but begin with phonemes of a matching word as compared to words with phonemes that differ from a matching word.

To account for the N200 effect in auditory sentence processing, it has been argued that a temporary set of candidates, which is built from the incoming phonemes, assists spoken word recognition (see van den Brink et al., 2001). This temporary activated set might be comparable to an expectancy set in priming paradigms. The enhanced right frontal negativity for incongruent words as compared to congruent words may reflect that congruent words are within this expectancy set, whereas incongruent words were not within this set. This is supported by a stronger behavioral facilitation for words than for pseudowords. In contrast, the left frontal negativity, which showed an enhanced amplitude for congruent pseudowords as compared to incongruent pseudowords, may reflect inhibitory processes if congruent words are in the expectancy set, but the target is classified as a pseudoword. In line with this interpretation, we found higher error rates for congruent pseudowords as compared to incongruent pseudowords in CMWP. 
The right frontal negativity for words was followed by an N400, which also showed an enhanced amplitude for incongruent as compared to congruent words. The amplitude of the N400 has been repeatedly reported to be sensitive to repetition of phonological or orthographic information. A reduced N400 amplitude has been found for targets in rhyming prime-target pairs (e.g., back-lack, Dumay et al., 2001; Praamstra, Meyer, \& Levelt, 1994; Rugg, 1984a, 1984b), for targets that share orthographic similarities with the prime (e.g., scan-scandal, Doyle, Rugg, \& Wells, 1996), and for targets in prime-target pairs with the same word stems (e.g., walk-walked, RodriguezFornells, Münte, \& Clahsen, 2002; Münte, Say, Clahsen, Schiltz, \& Kutas, 1999). Furthermore, the second presentation of words with or without intervening items is reported to reduce the N400 amplitude (e.g., Rugg, Doyle, \& Wells, 1995; Rugg \& Nieto-Vegas, 1999). In the present study, N400 effects were not found for pseudowords, indicating that the underlying processes were blocked if the target was not a word. These results point to an interpretation of the N400 as reflecting prime-target matching in phonological priming, which is executed after the target word has been correctly identified. If a relation between prime and target is detected, lexical decision is facilitated.

It remains to be explained why the N400 effect was more reliable in CMWP than in unimodal priming. One account is that there is a higher amount of information contained in an auditory than in a visual fragment. For example, syllable structure is marked in a spoken, but not in a visual, fragment. It has been shown that prosodic cues such as pitch, duration, and amplitude of syllables are exploited by the listener to guide spoken word recognition (e.g., Cooper et al., 2002; Cutler \& van Donselaar, 2001; Soto-Faraco et al., 2001). In a recent study, we were able to show that pitch contour is extracted already within the first $300 \mathrm{msec}$ of a spoken word (Friedrich, Alter \& Kotz, 2001). Furthermore, unlike letters in written language, phonemes are not discrete units of speech. Coarticulation often indicates an upcoming phoneme and this information is used by the listener (McMurray, Tannenhaus, \& Aslin, 2002; McQueen, Norris, \& Cutler, 1999; Marslen-Wilson \& Warren, 1994). Thus, there is more information in a spoken than in a written fragment, which predicts the upcoming word. Prime-target matching processes, possibly underlying the N400 effect, may rely on this additional information and thus work more efficiently for spoken than for visual fragments.

Unfortunately, we did not observe reliable effects of the number of simultaneously activated competitors in the present study. Therefore, we may ask whether the fragment length is an adequate manipulation to explore competition among activated candidates. It may not be possible to inhibit completely congruent targets, as no other activated candidate fits better with the incoming signal. Further research has to explore ERPs for targets which partially mismatch with the fragments. For those targets, we have to expect inhibition from such candidates that completely match with the fragment as demonstrated in a behavioral study by Soto-Faraco et al. (2001).

Taken together, the present experiments indicate that the combination of CMWP and the recording of ERPS provides a novel method to explore lexical identification independent of strategic processes. Facilitated lexical identification due to a modulated activation status of lexical entries appears to be reflected in the amplitude of the P350 in the ERP. Expectancy-based priming might be indexed by frontal negative ERP deflections, whereas prime-target matching appears to be reflected in the amplitude of the N400. The method described in this article allows us to address important issues in current neurophysiological research on correlates of word recognition. Furthermore, ERPs recorded in CMWP appear to be a powerful means to explore psycholinguistic hypotheses regarding the information that is used for lexical identification separately from information that is used for strategic operations.

\section{METHODS}

\section{Participants}

Twenty-four students (12 women, aged 18 to 30 years) participated in each of the two experiments. None of the subjects was tested in both experiments. All subjects were German speakers with normal hearing, normal or corrected-to-normal vision, and no history of neurological disorder. All subjects were right-handed according to the Edinburgh Handedness Inventory (Oldfield, 1971).

\section{Materials}

Fragments of different length (see Introduction) taken from 180 bisyllabic German words were presented as primes in Experiment 1. Words were spoken by a female German-speaker. Fragments were created by using a sound editor (CoolEdit v. 1.52, Syntrillium Software, Phoenix, AZ). None of the fragments was a complete German word. Short primes were on average $274 \mathrm{msec}$ long $(S D=133)$, medium fragments were $388 \mathrm{msec}$ long $(S D=88)$, and long fragments were 579 msec long $(S D=130)$. The auditory fragments were converted into letter strings presented in Experiment 2. Short fragments had on average 2.37 letters $(S D=0.82)$, medium fragments had 3.45 letters $(S D=0.86)$, and long fragments had 4.50 letters $(S D=0.91)$.

Using an online database of German ("Leipziger Wortschatz-Projekt") accessible via the Internet (http:// wortschatz.uni-leipzig.de) we analyzed how often the 
letter strings are beginnings of German words (excluding compounds). As we expected, the number of matching words decreased from the short to the medium fragments and was smallest for the long fragments. The beginning of on average 582.3 entries $(S D=926.0)$ in the database matched with the short fragments, 64.3 entries $(S D=138.2)$ matched with the medium fragments, and 2 entries $(S D=2.7)$ matched with the long fragments. Thus, although after hearing the long fragments, all subjects correctly guessed the original target words in the gating task, some of the fragments were ambiguous if presented visually. If syllable structure, stress pattern, and vowel quality are accounted for, on average, 1.1 word $(S D=0.3)$ matched with the long fragments. However, eight long fragments remained that matched two words. These analyses underline the fact that the spoken fragments were more informative than their written versions.

In both experiments, the original prime words were presented visually as congruent words. Congruent words were on average 5.95 letters long $(S D=1.02)$. Word frequency ranged between frequency class 9 and 20 according to the "Leipziger Wortschatz-Projekt." For each congruent word, a word with exactly the same word length and the same frequency, but different phonology and orthography, was selected as an incongruent word. For each congruent and each incongruent word, a pseudoword was created by interchanging the last letter (or the last two letters if both were consonants) between two words. For instance, Amboss and Balsam [balsam] were changed to the pseudowords Ambom and Balsass. Hence, half of the pseudowords was congruent with the preceding prime, whereas the other half was not.

\section{Differences between Targets}

In a control experiment, we presented the targets in a continuous lexical decision task. Twenty-four subjects that had not participated in one of the two priming experiments were tested. Behavioral and ERP responses were analyzed to explore differences for items presented as congruent and incongruent targets in the two priming experiments. Although the words were carefully controlled for frequency and number of letters, there was a material effect. Subjects made more errors for incongruent words $(14.1 \%, S D=4.3)$ than for congruent words $(6.9 \%, S D=3.1)\left[F_{1}(1,23)=53.32, p<.001, F_{2}(1,107)\right.$ $=10.21, p<.01]$. Furthermore, congruent words were responded to faster $(585 \mathrm{msec}, S D=68)$ than incongruent words $(596 \mathrm{msec}, S D=69)\left[F_{1}(1,23)=18.00\right.$, $\left.p<.001, F_{2}(1,107)=3.81, p=.05\right]$. Although this facilitation is in the same direction as in both priming experiments, the facilitation due to the material (11 msec faster and $7 \%$ less errors) was smaller than the smallest priming effect ( $47 \mathrm{msec}, 13 \%$ ). Besides the behavioral facilitation there was a reduced amplitude of the N400 in a time window between 400 and 600 msec for congruent words as compared to incongruent words $[F(1,23)=9.64, p<.01]$. Again, this effect was smaller $(1 \mu \mathrm{V})$ than the observed N400 effects in the priming conditions $(3.5 \mu \mathrm{V})$. Most importantly, however, there was no difference in the ERP amplitude within the time window of the P350 (200 to $300 \mathrm{msec}$ ). There were no effects in the material for pseudowords.

\section{Procedure}

The procedure in both experiments was identical, except for the prime's modality. Participants were comfortably seated in an electrically and acoustically shielded chamber in front of a computer screen with loudspeakers placed to the left and to the right side of the computer screen. Participants were instructed to keep their eyes on a fixation cross at the center of the screen. In Experiment 1, an auditory prime was presented, while the fixation cross remained on the screen. In Experiment 2, the fixation cross was replaced by a visual fragment with a duration of $200 \mathrm{msec}$. Immediately after the prime, a visual target was presented for $200 \mathrm{msec}$. The task was to respond as fast and as accurately as possible whether the target was a word or not. Note that in unimodal priming, superimposition of retinal images can be ruled out, because primes and targets differed in their length. Combined with the fixed central presentation of primes and targets, it was impossible that identical letters shared exactly the same position.

Presentation order of fragments of different length, congruent and incongruent targets, and words and pseudowords was counterbalanced. Half of the subjects gave "yes" responses with the thumb of the left hand and "no" responses with the thumb of the right hand; the other half had a reversed response pattern. Reaction times were measured from target onset.

The EEG was continuously recorded $(250 \mathrm{~Hz} / 22$ bit sampling rate; DC amplifier by Twente Medical Systems, Enschede, The Netherlands) from $58 \mathrm{Ag}-\mathrm{AgCl}$ electrodes, which were mounted in an elastic cap (Electro Cap International, Eaton, USA, 10-20 system). Two further electrodes were placed above the left and right mastoid. Those as well as the cap-mounted electrodes were referenced against the nose tip. Four further electrodes provided bipolar recordings of the horizontal and vertical electrooculogram (EOG). Impedances were kept below $5 \mathrm{k} \Omega$. Artifacts caused by facial and eye movements were rejected off-line when one of the EOG recordings exceeded $30 \mu \mathrm{V}$ within 200 msec. Furthermore, visual inspection of the raw EEG was carried out to eliminate drifts. ERPs were computed for the targets starting from the beginning of the visual presentation up to $600 \mathrm{msec}$ and with a 200-msec prestimulus baseline. Only correctly responded, artifact-free trials were included in the averaging procedure. 


\section{Data Analysis}

Behavioral data were subjected to three-way ANOVAs across subjects $\left(F_{1}\right)$ and across items $\left(F_{2}\right)$ including the factors Wordness (words vs. pseudowords), Congruency (congruent targets vs. incongruent targets), and Fragment length (short fragments vs. medium fragments vs. long fragments).

Four ROIs were defined for the ERP analyses. Each ROI included 11 electrode positions (left anterior: FP1, AF3, AF7, F3, F5, F7, F9, FC3, FC5, FT7, FT9; right anterior: FP2, AF4, AF8, F4, F6, F8, F10, FC4, FC6, FT8, FT10; left posterior: CP3, CP5, TP7, TP9, P3, P5, P7, P9, PO3, PO7, O1; right posterior: CP4, CP6, TP8, TP10, P4, P6, P8, P10, PO4, PO8, O2). Mean amplitudes were subjected to ANOVAs including the factors Wordness, Congruency, and Fragment length, as well as the factors Hemisphere (left vs. right) and Region (anterior vs. posterior). These ANOVAs were conducted across subjects. Significant interactions are only reported in case of significant post hoc comparisons. Because of different baselines (i.e., different auditory stimuli preceding the target), main effects of the factor Fragment length could not be analyzed in the ERPs.

\section{Acknowledgments}

The current work was supported by the Leibniz Science Prize awarded to A. D. Friederici and a grant from the German Research Foundation (DFG, FR 519/17-2). We thank Annett Schirmer and Shirley-Ann Rüschemeyer for helpful comments on former versions of the manuscript, and Christian Biemann for support with the "Leipziger Wortschatz-Projekt."

Reprint requests should be sent to Claudia K. Friedrich, Max Planck Institute of Cognitive Neuroscience, Stephanstrasse 1a, D-04103 Leipzig, Germany, or via e-mail: Claudia.Friedrich@ uni-konstanz.de.

\section{REFERENCES}

Andrews, S. (1989). Frequency and neighborhood effects on lexical access: Activation or search? Journal of Experimental Psychology: Learning, Memory and Cognition, 15, 802-814.

Coltheart, M., Davelaar, E., Jonasson, T. V., \& Besener, D. (1977). Access to the internal lexicon. In S. Domic (Ed.), Attention and performance (Vol. VI, pp. 535-555). Hillsdale, NJ: Erlbaum.

Connine, C. M., Titone, D., Deelmann, T., \& Blasko, D. (1997). Similarity mapping in spoken word recognition. Journal of Memory and Language, 37, 463-480.

Cooper, N., Cutler, A., \& Wales, R. (2002). Constraints of lexical stress on lexical access in English: Evidence from native and nonnative listeners. Language and Speech, 45, 207-228.

Cutler, A., \& Clifton, C. E. (1999). Comprehending spoken language: A blueprint of the listener. In C. M. Brown \& P. Hagoort (Eds.), The neurocognition of language (pp. 123-166). Oxford: Oxford University Press.
Cutler, A., \& van Donselaar, W. D. (2001). Voornaam is not (really) a homophone: Lexical prosody and lexical access in Dutch. Language and Speech, 44, 171-195.

Doyle, M. C., Rugg, M. D., \& Wells, T. (1996). A comparison of the electrophysiological effects of formal and repetition priming. Psychophysiology, 33, 132-147.

Dumay, N., Benraiss, A., Barriol, B., Colin, C., Radeau, M., \& Besson, M. (2001). Behavioral and electrophysiological study of phonological priming between bisyllabic spoken words. Journal of Cognitive Neuroscience, 13, 121-143.

Frauenfelder, U. H., \& Floccia, C. (1999). The recognition of spoken words. In A. D. Friederici (Ed.), Language comprebension: A biological perspective (pp. 1-40). Heidelberg: Springer-Verlag.

Friedrich, C. K., Alter, K., \& Kotz, S. A. (2001). An electrophysiological response to different pitch contours in words. NeuroReport, 12, 3189-3191.

Gow, D. W., \& Gordon, P. (1995). Lexical and prelexical influence on word segmentation: Evidence from priming. Journal of Experimental Psychology: Human Perception and Performance, 21, 344-359.

Grosjean, F. (1996). Gating. Language and Cognitive Processes, 11, 597-604.

Hagoort, P., \& Brown, C. M. (2000). ERP effects of listening to speech: Semantic ERP effects. Neuropsychologia, 38, 1518-1530.

Holcomb, P. J., Grainger, J., \& O'Rourke, T. (2002). An electrophysiological study of the effects of orthographic neighborhood size on printed word perception. Journal of Cognitive Neuroscience, 14, 981-950.

Keefe, D. E., \& Neely, J. H. (1990). Semantic priming in the pronunciation task: The role of prospective prime-generated expectancies. Memory and Cognition, 18, 289-298.

Kwantes, P. J., \& Mehwort, D. J. K. (1999). Evidence for sequential processing in visual word recognition. Journal of Experimental Psychology: Human Perception and Performance, 25, 376-381.

Lindell, A. K., Nicholls, M. E. R., \& Castles, A. E. (2003). The effects of orthographic uniqueness and deviation points on lexical decision: Evidence from unilateral and bilateral-redundant presentations. Quarterly Journal of Experimental Psychology, 65A, 287-307.

Marslen-Wilson, W. (1990). Activation, competition and frequency in lexical access. In G. T. M. Altmann (Ed.), Cognitive models of speech processing. Psycholinguistic and computational perspectives (pp.148-172). Cambridge: MIT Press.

Marslen-Wilson, W., \& Warren, P. (1994). Levels of perceptual representation and process in lexical access: Words, phonemes, and features. Psychological Review, 101, 653-675.

McMurray, B., Tanenhaus, M., \& Aslin, R. (2002). Gradient effects of within-category phonetic variation on lexical access. Cognition, 86, B33-B42.

McQueen, J. M., Norris, D., \& Cutler, A. (1994). Competition in spoken word recognition: Spotting words in other words. Journal of Experimental Psychology: Learning, Memory, and Cognition, 20, 621-638.

McQueen, J. M., Norris, D., \& Cutler, A. (1999). Lexical influence in phonetic decision making: Evidence from subcategorical mismatches. Journal of Experimental Psychology: Human Perception and Performance, 25, 1363-1389.

Münte, T. F., Say, T., Clahsen, H., Schiltz, K., \& Kutas, M. (1999). Decomposition of morphologically complex words in English: Evidence from event-related brain potentials. Cognitive Brain Research, 7, 241-253. 
Neely, J. (1991). Semantic priming effects in visual word recognition: A selective review of current findings and theories. In D. Besner \& G. Humphreys (Eds.), Basic processes in reading: Visual word recognition (pp. 264-337). Hillsdale, NJ: Erlbaum.

Neely, J. H., Keefe, D. E., \& Ross, K. L. (1989). Semantic priming in the lexical decision task: Roles of prospective prime-generated expectancies and retrospective semantic matching. Journal of Experimental Psychology: Learning, Memory, and Cognition, 15, 1003-1019.

Norris, D., McQueen, J. M., \& Cutler, A. (2002). Bias effects in facilitatory phonological priming. Memory and Cognition, 30, 399-411.

Oldfield, R. C. (1971). The assessment and analysis of handedness: The Edinburgh Inventory. Neuropsychologia, 9, 97-112.

Posner, M. I., \& Snyder, C. R. R. (1975). Facilitation and inhibition in the processing of signals. In P. M. A. Rabbitt \& S. Dornic (Eds.), Attention and performance $V$ (pp. 669-682). San Diego, CA: Academic Press.

Praamstra, P., Meyer, A. S., \& Levelt, W. J. M. (1994). Neurophysiological manifestations of phonological processing: Latency variation of a negative ERP component timelocked to phonological mismatch. Journal of Cognitive Neuroscience, 6, 204-219.

Pylkkänen, L., Stringfellow, A., \& Marantz, A. (2002). Neuromagnetic evidence for the timing of lexical activation: An MEG component sensitive to phonotactic probability but not to neighborhood density. Brain and Language, 81, 666-678.

Radeau, M., Morais, J., \& Dewier, A. (1989). Phonological priming in spoken word recognition: Task effects. Memory and Cognition, 17, 525-535.

Rodriguez-Fornells, A., Münte, T. F., \& Clahsen, H. (2002). Morphological priming in Spanish verb forms: An ERP repetition priming study. Journal of Cognitive Neuroscience, 14, 443-454.

Rugg, M. D. (1984a). Event-related brain potentials and the phonological processing of words and non-words. Neuropsychologia, 22, 435-443.

Rugg, M. D. (1984b). Event-related brain potentials in phonological matching tasks. Brain and Language, 23, 225-240.

Rugg, M. D., Doyle, M. C., \& Wells, T. (1995). Word and nonword repetition within- and across-modality: An event-related potential study. Journal of Cognitive Neuroscience, 7, 209-227.

Rugg, M. D., \& Nieto-Vegas, M. (1999). Modality-specific effects of immediate word repetition: Electrophysiological evidence. NeuroReport, 10, 2661-2664.

Soto-Faraco, S., Sebastian-Galles, N., \& Cutler, A. (2001). Segmental and suprasegmental mismatch in lexical access. Journal of Memory and Language, 45, 412-432.

Spinelli, E., Segui, J., \& Radeau, M. (2001). Phonological priming in spoken word recognition with bisyllabic targets. Language and Cognitive Processes, 16, 367-392.

van den Brink, D., Brown, C. M., \& Hagoort, P. (2001). Electrophysiological evidence for early contextual influences during spoken-word recognition: N200 versus N400 effects. Journal of Cognitive Neuroscience, 13, 967-985.

Vitevitch, M. S., \& Luce, P. A. (1999). Probabilistic phonotactics and neighborhood activation in spoken word recognition. Journal of Memory and Language, 40, 374-408.

Zwitserlood, P. (1989). The locus of the effects of sentential-semantic context in spoken-word processing. Cognition, 32, 25-64.

Zwitserlood, P. (1996). Form priming. Language and Cognitive Processes, 11, 589-596. 